\title{
Genetic selection system allowing monitoring of myofibrillogenesis in living cardiomyocytes derived from mouse embryonic stem cells
}

\author{
R. Bugorsky, ${ }^{1,2}$ J.C. Perriard, ${ }^{2}$ G. Vassalli ${ }^{1}$ \\ ${ }^{1}$ Department of Cardiology, Centre Hospitalier Universitaire Vaudois and Faculté de Biologie et \\ Médecine, University of Lausanne, Lausanne; ${ }^{2}$ Institute of Cell Biology, ETH-Zürich Hönggerberg, Zürich, \\ Switzerland
}

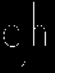

(C)2008 European Journal of Histochemistry

Embryonic stem (ES) cell-derived cardiomyocytes recapitulate cardiomyogenesis in vitro and are a potential source of cells for cardiac repair. However, this requires enrichment of mixed populations of differentiating ES cells into cardiomyocytes. Toward this goal, we have generated bicistronic vectors that express both the blasticidin $S$ deaminase (bsd) gene and a fusion protein consisting of either myosin light chain (MLC)-3f or human $\alpha$-actinin $2 \mathrm{~A}$ and enhanced green fluorescent protein (EGFP) under the transcriptional control of the $\alpha$-cardiac myosin heavy chain $(\alpha-\mathrm{MHC})$ promoter. Insertion of the DNase I-hypersensitive site (HS)-2 element from the $\beta$-globin locus control region, which has been shown to reduce transgene silencing in other cell systems, upstream of the transgene promoter enhanced MLC3f-EGFP gene expression levels in mouse ES cell lines. The $\alpha-\mathrm{MHC}-\alpha-$ actinin-EGFP, but not the $\alpha$-MHC-MLC3f-EGFP, construct resulted in the correct incorporation of the newly synthesized fusion protein at the Z-band of the sarcomeres in ES cellderived cardiomyocytes. Exposure of embryoid bodies to blasticidin S selected for a relatively pure population of cardiomyocytes within 3 days. Myofibrillogenesis could be monitored by fluorescence microscopy in living cells due to sarcomeric epitope tagging. Therefore, this genetic system permits the rapid selection of a relatively pure population of developing cardiomyocytes from a heterogeneous population of differentiating ES cells, simultaneously allowing monitoring of early myofibrillogenesis in the selected myocytes.

Key words: embryonic stem cells, cardiomyocytes, selection, differentiation, myofibrillogenesis, sarcomere

Correspondence: Jean-Claude Perriard,

Institute of Cell Biology, ETH-Zürich Hönggerberg,

8093 Zürich, Switzerland

E-mail: jean-claude.perriard@cell.biol.ethz.ch

Giuseppe Vassalli, Dept. of Cardiology, CHUV,

BH-10, Av. du Bugnon, 1011 Lausanne, Switzerland

E-mail: giuseppe.vassalli@chuv.ch

Paper accepted on December 20, 2007

European Journal of Histochemistry 2008; vol. 52 issue 1 (Jan-Mar): 1-10
D eath of cardiomyocytes, mainly due to acute or chronic myocardial ischemia, may lead to progressive heart failure. While pharmacological agents may slow down the progression of the disease, they ultimately fail to prevent it. Heart transplantation is the only definitive treatment for end-stage heart failure, but it suffers from major limitations including organ donor shortage, co-morbidity of immunosuppressive treatments, and chronic graft rejection. Over the past few years, cell transplantation has emerged as a potentially useful strategy for cardiac repair. Embryonic stem (ES) cells are an appealing candidate cell type for cell therapy approaches, as they can differentiate into derivatives of all three primary germ layers (endoderm, mesoderm and ectoderm) in vitro, including cardiomyocytes (Kehat et al., 2001; Xu et al., 2002; Fijnvandraat et al., 2003; Boheler et al., 2005). ES cells have been established as permanent lines of multipotent undifferentiated cells derived from the inner cell mass of blastocysts (Boheler et al., 2005). Cardiac differentiation in ES cell aggregates called embryoid bodies (EBs) is evidenced by the emergence of cell clusters showing spontaneous, rhythmic contractions. ES cell-derived cardiomyocytes resemble cardiomyocytes of the embryonic heart tube (Fijnvandraat et al., 2003). They express $\alpha$ and $\beta$-cardiac myosin heavy chain ( $M H C$ ) (Sanchez et al., 1991), myosin light chain (MLC) isoforms $2 a$ and 2v (Miller-Hance et al., 1993; Fijnvandraat et al., 2003), cardiac troponin I (cTnI) (Fijnvandraat et al., 2003), $\alpha$-tropomyosin (Muthuchamy et al., 1993), phospholamban (Ganim et al., 1992), sarcoendoplasmic reticulum calcium ATPase (SERCA) 2a (Fijnvandraat et al., 2003), atrial natriuretic factor (Miller-Hance et al., 1993), and type B natriuretic factor (Boer, 1994). They exhibit electrophysiologic features of atrial-like, ventricular-like, and conduction system-like cardiomyocytes (Kolossov et al., 2005). Therefore, ES cell-derived cardiomyocytes provide a unique model for studying structur- 
al and functional aspects of cardiomyogenic differentiation in vitro.

Because pluripotent ES cells differentiate into many different cell types, enrichment of mixed populations of differentiating ES cells into cardiomyocytes is required. In fact, transplantation of pluripotent ES cells can induce tumor formation (teratoma) (Erdo et al., 2003). Earlier genetic selection systems have used two different approaches to purify ES cell-derived cardiomyocytes. Klug et al. (1996) generated genetically modified totipotent mouse ES cells that expressed an aminoglycoside resistance gene under the transcriptional control of the $\alpha$-cardiac MHC ( $\alpha$-MHC) promoter. Transgenic ES cell-derived cardiomyocytes enriched with G418 after in vitro differentiation were injected into mouse hearts where they were detectable in vivo 7 weeks thereafter. Several studies have used a reporter gene approach. Kolossov et al. generated stably transfected ES cells expressing the green fluorescent protein (GFP) gene under control of the human cardiac $\alpha$-actin promoter (Kolossov et al., 1998) or enhanced GFP (EGFP) under control of the $\alpha-$ MHC promoter (Kolossov et al., 2005). EGFP expression in cardiac precursors was observed as early as 7 days of development, even before the appearance of spontaneous contractions. Müller et al. (2000) and Meyer et al. (2000) purified ES cell-derived cardiomyocytes from EBs stably transfected with the EGFP reporter gene or its cyan variant (ECFP) under control of the MLC-2v promoter. This promoter was switched on as early as day 7, as evidenced by the appearance of fluorescent cells, followed by that of spontaneous beating activity in all regions containing such cells by day 8 . Moore et al. (2004) used a P19C16 GFP reporter line to quantify cardiomyocyte differentiation of stem cells. Hidaka et al. (2003) developed an in vitro culture system to track cell lineages positive for the cardiac transcription factor NkX-2.5 during mouse ES cell differentiation using GFP as a reporter. Huber et al. (2007) generated stable transgenic human ES cell lines using lentiviral vectors that express EGFP under control of the MLC$2 v$ promoter. ES cell-derived $\mathrm{EGFP}^{+}$cardiomyocytes purified by fluorescence-activated cell sorting (FACS) formed stable cell grafts in vivo after intramyocardial cell injection.

The two selection modalities - drug resistance and reporter genes - have distinct advantages and limitations. While drug selection results in relatively pure populations of ES cell-derived cardiomyocytes, it does not allow direct visualization of the selected cardiomyocytes, nor of myofibrillogenesis taking place in these cells. This requires cell fixation, permeabilization, and immunostaining of myofibrillar proteins, which precludes longitudinal analysis of different stages of myofibrillogenesis in viable cells. Conversely, ES cell-derived cardiomyocytes that express a reporter gene can be readily identified and purified by FACS, but this requires enzymatic dissociation of cell aggregates to obtain a population of individual cells. Although ES cell-derived cardiomyocytes can recover after the FACS procedure and reestablish cell-cell contacts and sarcomeres in culture, this manipulation perturbs the regular unfolding of cell differentiation and myofibrillogenesis in vitro. Hence, combining the drug resistance and the reporter gene approach may be advantageous. Toward this goal, we generated bicistronic vectors that express both the blasticidin S deaminase ( $b s d$ ) gene from Aspergillus terreus (Kimura et al., 1994) and a fusion protein consisting of a sarcomeric protein - the MLC isoform MLC3f or the human $\alpha$ actinin $2 \mathrm{~A}$ isoform - and EGFP under the transcriptional control of the $\alpha \mathrm{MHC}$ promoter. Additional cardiac-specific promoters were also tested. Blasticidin S treatment of EBs from transgenic ES cell lines resulted in a relatively pure population of cardiomyocytes after 3 days of selection. Correct localization of the $\alpha$-actinin-EGFP fusion protein at the Z-band of the sarcomeres allowed monitoring of myofibrillogenesis in differentiating cardiomyocytes by vital fluorescence microscopy.

\section{Materials and Methods}

\section{Gene expression vectors}

The principal vectors used in the present study were derived from the bicistronic SM18.2 expression plasmid (7165 bp) that contains a MLC3f (bp 942-2159)-EGFP fusion cassette (Auerbach et al., 1997) driven by the cytomegalovirus-immediate/ early (CMV-IE) promoter, the Internal Ribosome Entry Site (IRES) element (starting at bp 2187; derived from pIRES, Clontech Laboratories, Palo Alto, CA, USA), the bsd gene (inserted $3^{\prime}$ of the IRES at bp 2772-3150; derived from pCMV/Bsd, Invitrogen, Basel, Switzerland), and a puromycin resistance $\left(\right.$ Puro $\left.^{R}\right)$ cassette (bp 4126-4706) driven by the phosphoglycerate kinase (PGK) promoter 
(Figure 1). The $\alpha$-MHC promoter was subcloned into the SM18.2 vector as follows: the $5.5-\mathrm{kb} \alpha$ MHC promoter fragment (Gulick et al., 1991) comprising of $4.5 \mathrm{~kb}$ of $5^{\prime}$ flanking sequence and 1 $\mathrm{kb}$ of the gene encompassing exons 1 through 3 up to, but not including, the initiation codon was amplified from the $\alpha \mathrm{MHC}$-clone26 plasmid (gift of Dr. J. Gulick, Children's Hospital, Cincinnati, OH, USA). Insertion of the M/uI/MfeI-digested PCR-amplification fragment (primers: Table 1) from $\alpha \mathrm{MHC}$ clone26 into MIUI/ECoRI-digested SMI8.2 released the CMV-IE promoter fragment (bp 228935). E. coli Top10 (Invitrogen) were transformed with the ligation product, yielding the $\alpha-\mathrm{MHC}$ MLC3f-EGFP-IRES-BSD expression plasmid. The 750-bp MLC-2v promoter fragment including 3 copies of the HF-1, HF-2 and HF-3 upstream elements that enhance promoter activity and cardiac specificity (Jin et al., 1995) was amplified from the MLCp.luc plasmid (gift of Dr. Y. Jin, University of Manitoba, Winnipeg, Canada) by PCR. The MluI/MfeI-digested amplification fragment from MLCp.luc was subcloned into SM18.2, yielding the MLC2v-MLC3f-EGFP-IRES-BSD construct. The 2.6-kb Na${ }^{+} / \mathrm{Ca}^{+}{ }^{+}$exchanger 1 (NCX1) promoter sequence was amplified from the pGL2-Basic Vector+2.6 Kb NCX1 promoter vector (Müller et al., 2002) (gift of Dr. J. G. Müller, Medical University of South Carolina, Charleston, SC, USA). The MluI/ECoRI-digested PCR fragment was subcloned into SM18.2, yielding the NCXMLC3f-EGFP-IRES-BSD construct. The 463-bp human cTnI promoter fragment was amplified from the -463troponinI gene construct (Bhavsar et al., 2000) (gift of Dr. P. Barton, Imperial College School of Medicine, London, UK). The MIUI/EcoRI-

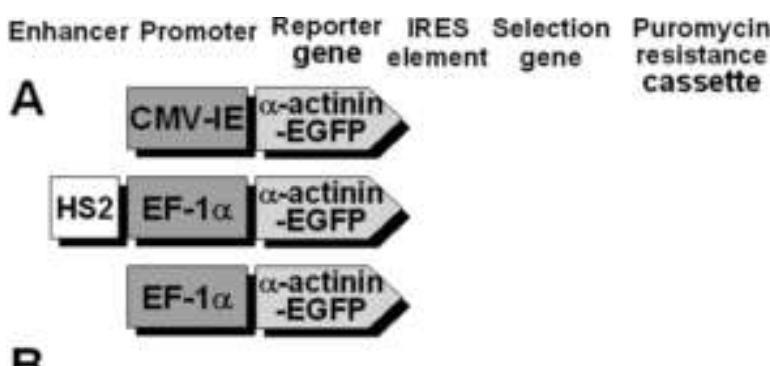

B

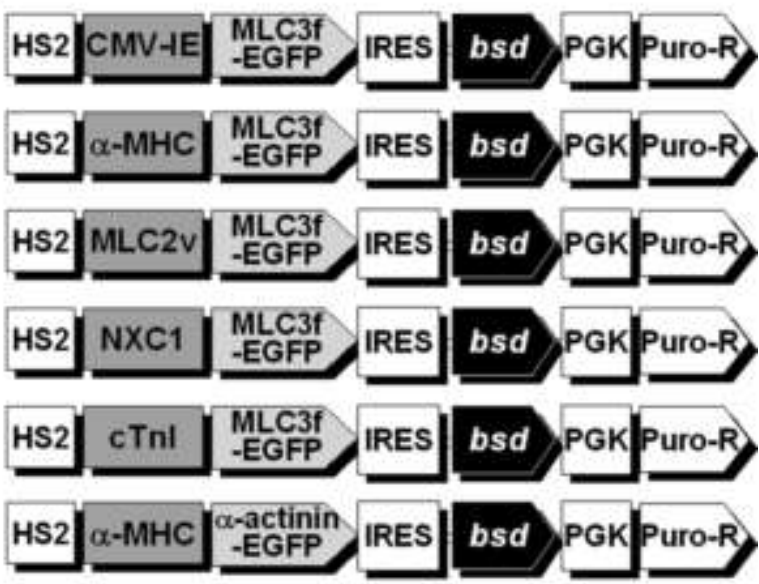

Figure 1. Schematics of the principal expression vectors used in the present study. Boxes depict functional elements as indicated on top of figure. (A) Vectors used for analysis of the effect of the HS2 element on $\alpha$-actinin-EGFP expression in ES cell lines. (B) Bicistronic HS2 element-containing vectors used in rat cardiomyocytes, COS cells, and ES cell lines (abbreviations: see text).

cut amplification fragment was subcloned into SM18.2, yielding the cTnI-MLC3f-EGFP-IRESBSD construct. The human $\alpha$-actinin $2 \mathrm{~A}$ isoform gene was amplified by PCR from pET6-ACTN2 (Young et al., 1998) (gift of Dr. M. Gautel, The Randall Centre, King's College, London, UK). The EcoRI/NotI MLC3f-EGFP fragment (1.2-kb) of $\alpha-M H C-M L C 3 f-E G F P-I R E S-B S D$ was replaced

Table 1. PCR primers used for amplification of DNA fragments for molecular cloning: lox66-MHC.up/lox66-MHC.lo for the $\alpha$-MHC promoter fragment; HumTropLox66.s/ HumTropLox66.as for the cTnl promoter fragment; MLC2vLox66.s/MLC2vLox66.as for the MLC-2v promoter fragment; NCX-upp/NCX-low for the NCX1 promoter fragment; HS2ext-s/HS2ext-as for amplification of the 523 bp fragment of the HS2 element from genomic DNA; HS2Mlu.s/HS2Mlu.as for the HS2-EF1 $\alpha$-EGFP fragment.

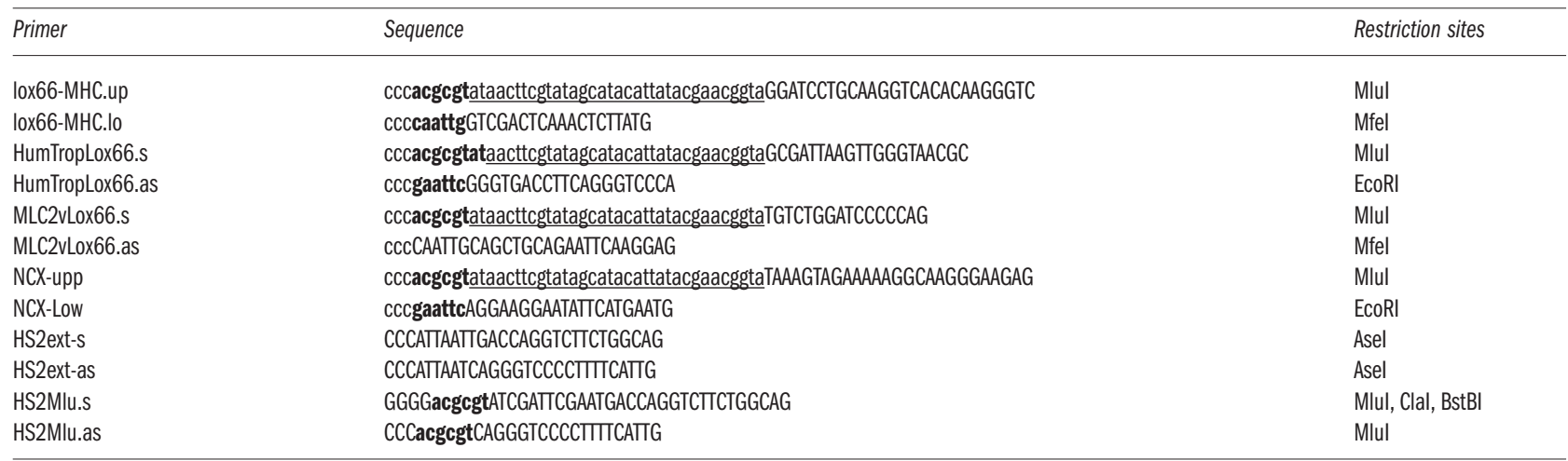


with the $\alpha$-actinin-EGFP fragment (Dätwyler et al., 2001), yielding the $\alpha$-MHC- $\alpha$-actinin-EGFPIRES-BSD construct. The 523-bp DNase I-hypersensitive site (HS)-2 element (accession number: S73747.1) from the $\beta$-globin Locus Control Region (LCR) (Walters et al., 1996; Bulger et al., 1999; Grosveld, 1999) was amplified from mouse genomic DNA by PCR (primers: see Table 1), digested with MIUI, and subcloned into the SM13.4 and FS2.2 vectors that contain an expression cassette for the $\alpha$-actinin-EGFP fusion protein driven by the CMV-IE promoter and the 475-bp human elongation factor (EF)-1 $\alpha$ promoter (Chung et al., 2002), respectively, yielding the HS2-CMV- $\alpha$-actininEGFP and HS2-EF1- $\alpha$-actinin-EGFP expression constructs. Finally, the HS2 fragment was also inserted upstream of the respective cardiac-specific promoters in bicistronic expression constructs.

\section{Stable transfection of mouse ES cells}

ES cells from the RI cell line (Nagy et al., 1993) (gift of Dr. A. Nagy, Mount Sinai Hospital, Toronto, Canada) were used at P17 and cultured in Knockout Dulbecco's modified Eagle's medium (DMEM) supplemented with L-glutamin, nonessential amino acids, $1 \%$ sodium pyruvate, $15 \%$ Knock-out serum replacement (all from Gibco Laboratories, Grand Island, NY, USA), $1 \mathrm{mmol} / \mathrm{L} \beta$-mercaptoethanol (Sigma, St. Louis, MO, USA), and leukemia inhibitory factor (LIF)-conditioned medium $(0.4 \%)$, essentially as described (Smith, 1992). Electroporation, puromycin selection, and propagation of clones was performed according to standard protocols (Kolossov et al., 1998). Briefly, ES cells were trypsinized, resuspended in PBS, and diluted to a final concentration of $7 \times 10^{5}$ cells $/ \mathrm{mL}$. The cell suspension $(0.8 \mathrm{~mL})$ was mixed with 10 $\mu \mathrm{g}$ of vector DNA linearized with M/uI and electroporated at $250 \mathrm{~V}$. Cells were plated onto 100$\mathrm{mm}$ plates in $13 \mathrm{ml}$ ES cell medium. After $48 \mathrm{~h}, 3$ $\mu \mathrm{g} / \mathrm{mL}$ puromycin (InvivoGen; San Diego, CA, USA) was added. Stably transfected cell clones ( $=25$ for each transfection vector tested) were monitored daily for appearance of green fluorescent cell clusters under a fluorescence microscope.

\section{Transient transfection of neonatal rat cardiomyocytes}

Newborn rat hearts were dissected, ventricles were minced, and tissue fragments were digested with collagenase (Worthington Biochemicals, Lakewood, NJ, USA) and pancreatin (Gibco), fol- lowed by purification on a Percoll density step gradient, and cultured essentially as described (Sen et al., 1988). Cells ( $4 \times 10^{5}$ cells/dish) were plated on fibronectin $(10 \mu \mathrm{g} / \mathrm{mL})$-coated dishes in $67 \%$ DMEM, 17\% M199, 4 mmol L-glutamine (Amimed, Basel, Switzerland), 10\% horse serum (Gibco), 5\% fetal calf serum (FCS; PAA Laboratories, Parker Ford, PA, USA), and 1\% penicillin/streptomycin (Gibco). After $24 \mathrm{~h}$, medium was replaced with maintenance medium $(78 \%$ DMEM, 20\% M199, 1\% horse serum, 4 mmol Lglutamine, $1 \%$ penicillin/streptomycin, and $0.1 \mathrm{mM}$ phenylephrine; Sigma). For transfection, $1 \mu \mathrm{g}$ of vector DNA and $4 \mu \mathrm{L}$ of Escort III (Sigma) were diluted in $100 \mu \mathrm{L}$ of OptiMEM (Gibco). The transfection mix was added drop by drop to cell cultures according to the manufacturer's instructions. After cell incubation at $37^{\circ} \mathrm{C}$ for $4 \mathrm{~h}$, transfection medium was replaced with maintenance medium.

\section{Transfection of COS-1 cells}

COS-1 cells were obtained from the American Type Culture Collection (Manassas, VA, USA) and grown at $37^{\circ} \mathrm{C}$ under $5 \% \mathrm{CO}_{2}$ in $89 \%$ Iscove's MEM (Amimed), $4 \mathrm{mmol} / \mathrm{L}$ glutamine, $1 \%$ penicillin/ streptomycin, $10 \%$ FCS (PAA Laboratories), and 1 $\mathrm{mmol} / \mathrm{L} \beta$-mercaptoethanol. A mixture of $97 \mu \mathrm{L}$ OptiMEM and $3 \mu$ L FuGENE 6 Transfection Reagent (Roche, Basel, Switzerland) was used for transfection. DNA ( $1 \mu \mathrm{g}$ ) was added to the transfection mixture, which was incubated for $15 \mathrm{~min}$ at RT, and then added to COS-1 cells according to the manufacturer's instructions. Transfected cells were incubated in the above medium under $10 \% \mathrm{CO}_{2}$ for 2 days.

\section{Functional analysis of the HS2 element in trans- genic ES cell lines}

To assess the effect of the HS2 element on $\alpha$ actinin-EGFP fusion protein expression, ES cells were stably transfected with either the CMV- $\alpha$ actinin-EGFP or the EFl- $\alpha$-actinin-EGFP vector, with or without the insertion of the HS2 element upstream of the transgene promoter. Flow cytometric analysis of EGFP expression was performed using a dual laser FACSCalibur ${ }^{\circledR}$ (Becton Dickinson, Germany). ES cells were trypsinized and resuspended to a concentration of $5 \times 10^{5}$ cells $/ \mathrm{mL}$ in PBS shortly before analysis. FITC bandpass filter was used to measure EGFP fluorescence intensity excited with the $488 \mathrm{~nm}$ line of an argon ion 
laser. Detector settings were adjusted with nontransfected ES cells of the cell line R1. Each analysis included $10^{4}$ cells/clone $(n=5$ clones/vector type). Data were recorded with the CellQuest ${ }^{\mathrm{TM}}$ acquisition software (Becton Dickinson).

\section{ES cell differentiation and selection of cardio- myocytes}

To induce ES cell differentiation, ES cells $(16,000 / \mathrm{mL})$ were suspended in Knock-out DMEM medium supplemented with $4 \mathrm{mmol}$ L-glutamine, nonessential amino acids, $1 \%$ sodium pyruvate, $15 \% \mathrm{FCS}$, and $1 \mathrm{mmol} / \mathrm{L} \beta$-mercaptoethanol. Hanging drops were formed by plating $30 \mu \mathrm{L}$ of cell suspension on the lids of $150-\mathrm{mm}$ bacteriological dishes. After cell incubation at $37^{\circ} \mathrm{C}$ under $5 \% \mathrm{CO}_{2}$ for $48 \mathrm{~h}$, EBs were formed. They were collected in $15 \mathrm{~mL}$ differentiation medium, cultured in suspension in bacteriological dishes for 2 days, and plated onto 100 and 35-mm tissue culture dishes coated with $0.1 \%$ gelatin (Fluka, Milan, Italy) at a density of 50 and 10 EBs per dish, respectively. The time point of ES cell plating was defined as day 0 . EBs were monitored daily for appearance of spontaneously and rhythmically contracting myocytes under an inverted light microscope. To document beating activity, phase-contrast images were acquired at $5 \mathrm{sec}$ intervals using a Hamamatsu color chilled 3CCD camera connected to the inverted microscope, and fast cell motion was visualized by pixel intensity subtraction analysis of sequential images with Photoshop 7.0 (Adobe Systems, San Jose, CA, USA). After 10 days in differentiation medium, blasticidin S (InvivoGen) was added to cell cultures at varying concentrations ( $7-44 \mu \mathrm{g} / \mathrm{mL}$; previous experiments showed no effect at $<7 \mu \mathrm{g} / \mathrm{mL}$ ). After 3 days of selection, 10-20 EBs were washed with PBS and then dissociated to a single cell suspension by trypsin treatment for 2-3 min. Then, 1 $\mathrm{ml}$ of DME $+20 \%$ FCS was added to the single cell suspension. After centrifugation (1000 rpm) for $5 \mathrm{~min}$, cells were resuspended in $0.5-1.0 \mathrm{~mL}$ of PBS containing $\mathrm{Ca}^{2+}(1 \mathrm{mM})$ and $\mathrm{Mg}^{2+}(0.5 \mathrm{mM})$, plated on gelatin-coated dishes, cultured overnight, and immunostained with mouse monoclonal $(\mathrm{Mm})$ anti-M HC (Covance, Berkeley, CA, USA) or striated muscle $\mathrm{CHI}$ tropomyosin-specific antibodies (Sigma). In separate experiments, blasticidin selection was extended up to 10 days to assess toxicity.

\section{Immunocytochemistry}

Transfected neonatal rat cardiomyocytes were washed with PBS, fixed with $4 \%$ paraformaldehyde (PFA) in PBS ( $p H$ 7.4) for $10 \mathrm{~min}$, then washed with $P B S$, incubated in $0.1 \mathrm{M}$ glycine in PBS for $5 \mathrm{~min}$, and permeabilized with $0.2 \%$ Triton $X-100$ in PBS for $10 \mathrm{~min}$, and blocked with $5 \%$ normal goat serum (NGS) in $1 \%$ bovine serum albumin (BSA) in PBS for $30 \mathrm{~min}$. They were stained with $\mathrm{Mm}$ anti-rabbit skeletal $\alpha$-actinin (sarcomeric; EA-53, Sigma; 1:500) antibody in $\mathrm{PBS} / 1 \% \mathrm{BSA}$ at $4^{\circ} \mathrm{C}$ overnight. For immunofluorescence, an appropriate Cy3-conjugated antimouse secondary antibody (Jackson Immuno Research, Hamburg, Germany; 1:200) was used, as described (Ehler et al., 1999). EBs before and after blasticidin selection were stained with anti-sarcomeric $\alpha$-actinin (1:500) and rabbit polyclonal (Rp) anti-pan-cadherin (C3678, Sigma ; $1: 200$ ) antibodies in $\mathrm{PBS} / 1 \% \mathrm{BSA}$ at $4^{\circ} \mathrm{C}$ overnight. For immunofluorescence, appropriate Cy3-conjugated anti-mouse (Jackson ImmunoResearch; 1:500) and FITC-conjugated anti-rabbit (Cappel, Organon Teknika, Pfäffikon, Switzerland; 1:500) secondary antibodies were used, as described (Ehler et al., 1999). Controls for antibody specificity included omitting primary antibodies, replacing primary antibodies with isotypematched control antibodies, and staining with each of the primary antibodies followed by the reciprocal secondary antibody. Actin was visualized using Alexa Fluor-633-conjugated phalloidin (Molecular Probes, Eugene, OR, USA; 1:100), as described (Ehler et al., 1999). ES cell-derived cardiomyocytes were stained with the monoclonal B4 antibody against myomesin (Grove et al., 1984), which was raised in our laboratory. Specimens were analyzed by confocal laser scanning microscopy.

\section{Confocal laser scanning microscopy}

The imaging system consisted of a Leica inverted microscope DM IRB/E, a Leica true confocal scanner TCS SPI (Leica Microsystems AG, Glattbruggg, Switzerland), and a Fujitsu-Siemens workstation (Fujitsu Siemens computer Bv., Regensdorf, Switzerland). The images were recorded using a Leica PL APO×63/1.4 oil immersion objective. The system was equipped with $\mathrm{Ar}$ and $\mathrm{He} / \mathrm{Ne}$ lasers. Image processing was done using Imaris $^{\circledR} 4.0$ (Bitplane AG, Zurich, Switzerland). 


\section{Results}

\section{The HS2 element enhances EGFP expression in transgenic ES cell lines}

The effect of the HS2 element on transgene expression in ES cell lines was assessed using an $\alpha$ actinin-EGFP cassette driven by either the CMVIE or the EF-1 $\alpha$ promoter, with or without an upstream HS2 element. EGFP expression was detectable by FACS in ES cell lines containing the $E F-1 \alpha$, but not the CMV-IE, promoter-driven $\alpha$ actinin-EGFP cassette. In the presence of the HS2 element, EGFP expression was detectable in 5 of 5 clones, compared with only 2 of 5 clones in its absence (Figure 2A). In EGFP ${ }^{+}$clones, presence of the HS2 element within the expression construct was associated with a approximately 10-fold increase in the number of $\mathrm{EGFP}^{+}$cells and a 5 to 10 -fold increase in mean fluorescence intensity (MFI) (Figure 2B).

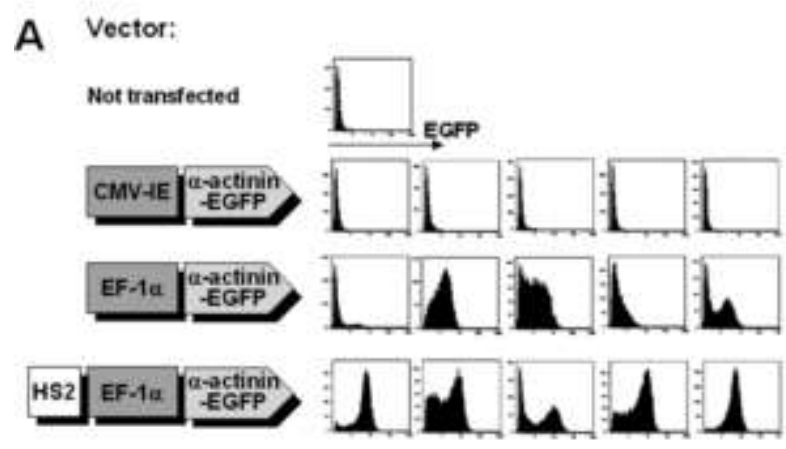

B

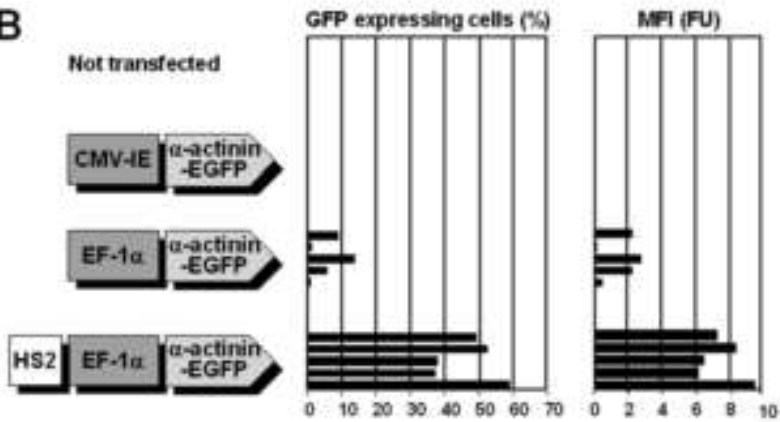

Figure 2. Effects of the HS2 enhancer element on $\alpha$-actininEGFP expression in stably transfected ES cells. (A) Flow cytometry histograms showing EGFP expression in $\mathbf{5}$ clones for each vector tested. (B) Numbers of EGFP' cells (\%) and mean fluorescence intensity (MFI; FU, arbitrary fluorescence units). Horizontal bars indicate individual clones.

\section{Assessment of cardiac-specific MLC3f- or $\alpha$-actinin-EGFP expression in neonatal rat car- diomyocytes}

We generated bicistronic vectors containing the HS2 element, a cardiac-specific promoter (the $\alpha$ MHC, MLC-2v, NCXI or the cTnI promoter), either the MLC3f-EGFP or $\alpha$-actinin-EGFP fusion gene, the IRES element, and the bsd cassette (Figure 1 ). These vectors were first tested in transiently transfected neonatal rat cardiomyocytes and COS-1 cells (the latter as a control for tissue specificity). MLC3f-EGFP gene expression driven by each of the four cardiac-specific promoters was detectable by fluorescence microscopy in transfected neonatal rat cardiomyocytes (Figure 3 ), but not in COS-1 cells (Figure 4). While vectors containing the MLC-2v, NCXI or the CTnI promoter resulted in the correct localization of the MLC3f-EGFP fusion protein to the Z-band of the sarcomeres, the HS2- $\alpha-M H C-M L C 3 f-E G F P-$

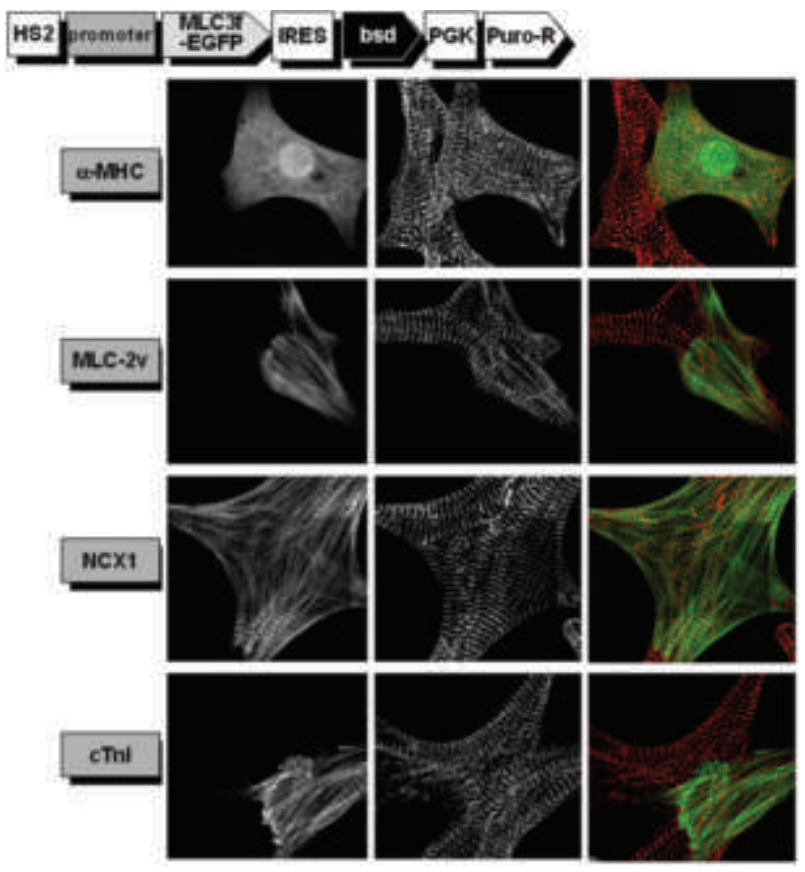

Figure 3. Confocal laser scanning micrographs of neonatal rat cardiomyocytes transiently transfected with bicistronic vectors expressing the bsd gene and a MLC3f-EGFP cassette driven by the indicated cardiac-specific promoters (see Figure 1). Columns: left, green fluorescence microscopy; middle, sarcomeric $\alpha$-actinin immunostaining; right: color merge images (EGFP, green; $\alpha$-actinin, red). Correct incorporation of MLC3f-EGFP at the Z-band of the sarcomeres was observed with the MLC-2v, NCX1 and cTnl promoters, but not the $\alpha$ MHC promoter (diffuse intracellular distribution of EGFP). 


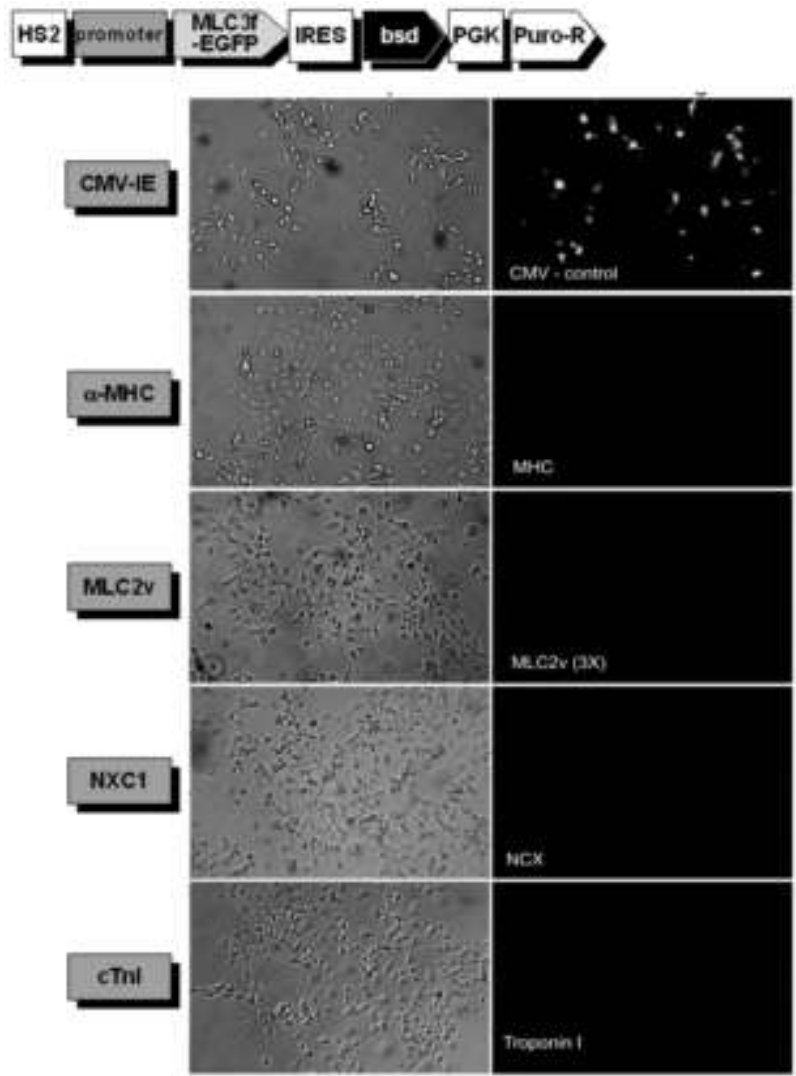

Figure 4. Confocal laser scanning micrographs of COS-1 cells transiently transfected with bicistronic vectors expressing the bsd gene and a MLC3f-EGFP cassette driven by the indicated cardiac-specific promoters (see Figure 1). A CMV-IE promotercontaining vector was used as a control. Columns: left, phasecontrast microscopy; right, fluorescence microscopy. No EGFP expression was observed using cardiac-specific promoters.

IRES-BSD vector resulted in a diffuse intracellular distribution of the fusion protein (Figure 3 ). This problem was not observed using the $\alpha$-actininEGFP cassette driven by the $\alpha$-MHC promoter, which resulted in the correct epitope tagging in ES cell lines.

\section{Establishment and specificity of $\alpha-M H C$ promoter- driven MLC3f- or $\alpha$-actinin-EGFP expression in ES cell lines}

Transgenic ES cell lines wexre generated by stable transfection with the HS2- $\alpha-M H C-M L C 3 f-$ EGFP-IRES-BSD or HS2- $\alpha$-MHC- $\alpha$-actininEGFP-IRES-BSD vectors. Cell clusters in EBs exhibiting bright green fluorescence were detectable around days 7-8 by fluorescence microscopy in 3 of 25 clones tested for each of the two vectors. The appearance of fluorescent cell clusters preceded the onset of spontaneous con-
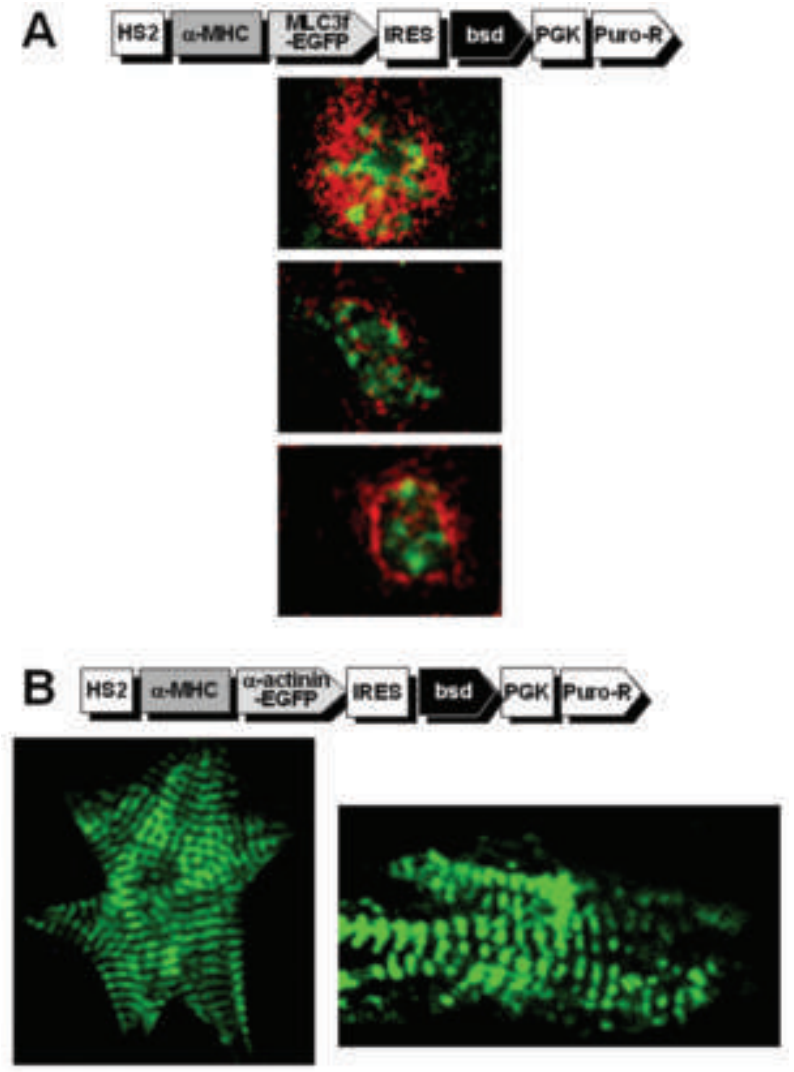

Figure 5. Vital fluorescence microscopy of EBs. (A) Three different HS2- $\alpha$-MHC-MLC3f-EGFP-IRES-BSD ES cell lines showing green fluorescent regions. Pixel intensity subtraction analysis depicts spontaneously contracting regions in red. All green spots were co-localized with red areas. (B) High-magnification, vital fluorescence micrographs of HS2- $\alpha$-MHC- $\alpha$-actinin-EGFP-IRES-BSD ES cell-derived cardiomyocytes showing sarcomeric tagging.

tractions in the same regions on days 8-9 (Figure 5A). No green fluorescent area was located outside contracting regions. Again, the $\alpha$-actinin-EGFP, unlike the MLC3f-EGFP, fusion protein was correctly localized to the Z-line of sarcomeres (Figure $5 B$ ). No green fluorescence was detectable in ES cell clones generated with MLC-2v, NCXI or CTnI promoter-containing vectors ( $n=25$ clones/vector).

\section{Blasticidin S selection and characterization of ES cell-derived cardiomyocytes}

ES cell-derived cardiomyocytes in EBs stained positive for sarcomeric $\alpha$-actinin and pan-cadherin (Figure 6). Pan-cadherin delineated the whole perimeter of cell membranes rather than being concentrated in intercalated disks, which is characteristic for embryonic cardiomyocytes. In the parent RI ES cell line, $12 \mu \mathrm{g} / \mathrm{mL}$ blasticidin selection started on day 10 caused massive cell death by 48 

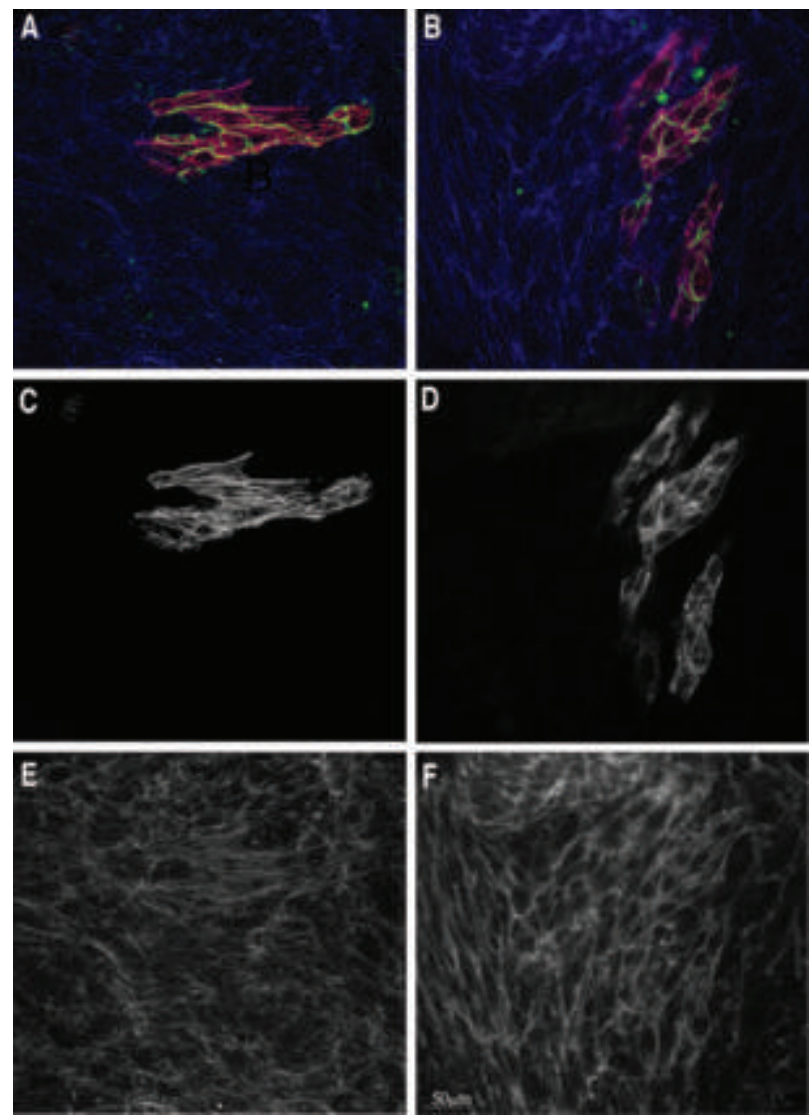

Figure 6. Confocal laser scanning micrographs images of EBs before blasticidin selection. Left and right columns show two examples of ES cell-derived cardiomyocyte clusters. Upper row: color merge images (immunostaining for pan-cadherin, green; sarcomeric $\alpha$-actinin, red; phalloidin, blue); middle row: $\alpha$-actinin; lower row: phalloidin.

h. Virtually all cells were dead and detached from the plates by $72 \mathrm{~h}$. Treatment with $34 \mu \mathrm{g} / \mathrm{mL}$ blasticidin caused detachment of virtually all of the

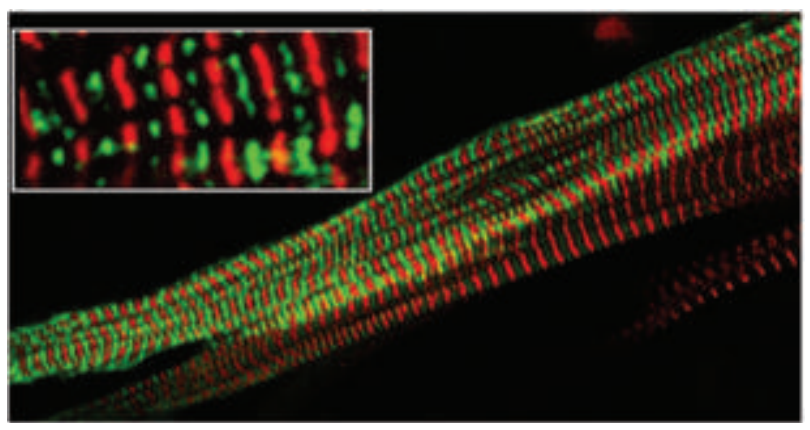

Figure 8. Confocal laser scanning micrograph of selected, cultured HS2- $\alpha-M H C-\alpha-a c t i n i n-E G F P-I R E S-B S D$ cardiomyocytes. EGFP, green; myomesin immunostaining, red. Insert: high-magnification; the $\alpha$-actinin-EGFP fusion protein is located at the Zline of sarcomeres.
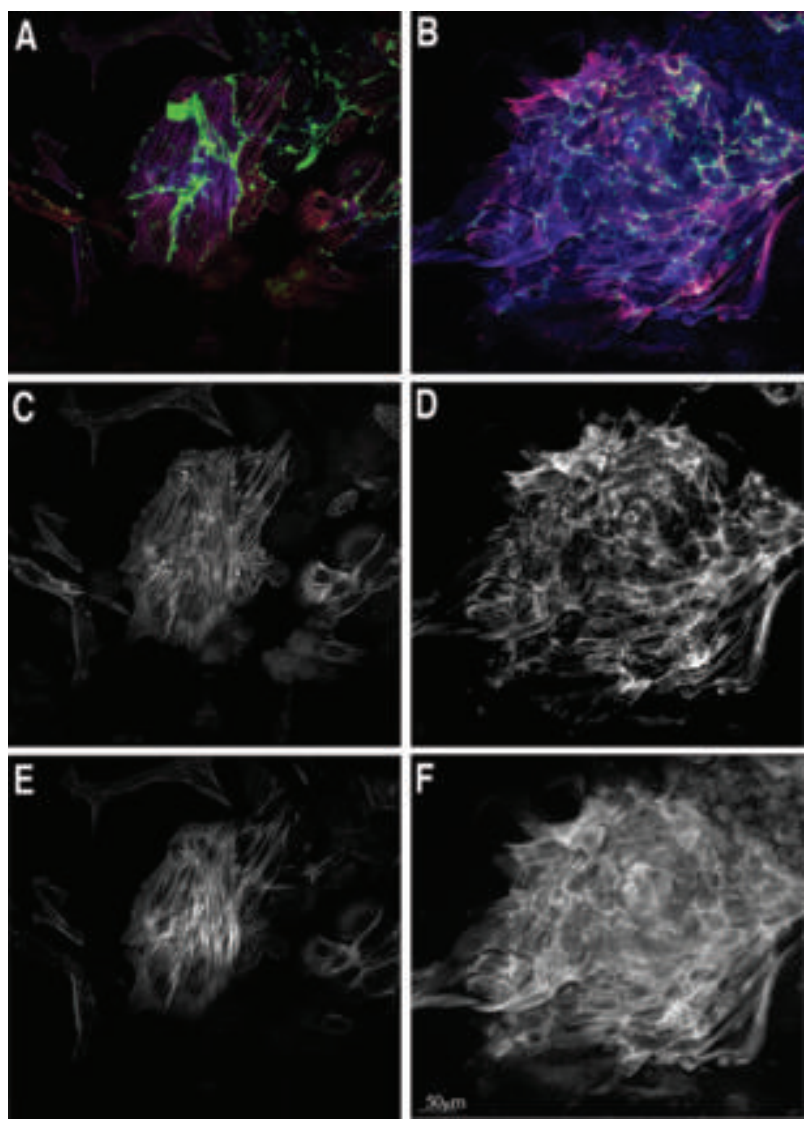

Figure 7. Confocal laser scanning micrographs images of EBs after 3 days of blasticidin selection. Left and right columns show two examples of ES cell-derived cardiomyocyte clusters. Upper row: color merge images (pan-cadherin, green; sarcomeric $\alpha$-actinin, red; phalloidin, blue); middle row: $\alpha$-actinin; lower row: phalloidin. Compared to Figure 6, phalloidin-positive but $\alpha$ actinin-negative regions surrounding $\alpha$-actinin-positive areas have disappeared.

cells by 48 h. In EBs containing green fluorescent beating regions, virtually all cells outside those regions detached after treatment with $34 \mu \mathrm{g} / \mathrm{mL}$ blasticidin for $48 \mathrm{~h}$, followed by $12 \mu \mathrm{g} / \mathrm{mL}$ for 24 h. Surviving cell clusters stained positive for sarcomeric $\alpha$-actinin and pan-cadherin (Figure 7 ). They survived treatment with $44 \mu \mathrm{g} / \mathrm{mL}$ blasticidin during 10 days with no apparent toxicity. FACS analysis of cells dissociated from EBs before selection showed $5.1 \pm 3.4 \%$ and $4.5 \pm 2.2 \%$ of cells staining positive for $\alpha-\mathrm{MHC}$ and tropomyosin, respectively, as compared with $>98 \%$ after selection. Selected cardiomyocytes grew normally in culture and exhibited correct incorporation of the newly synthesized $\alpha$-actinin-EGFP fusion protein at the Z-band of the sarcomeres (Figure 8). 


\section{Discussion}

ES cells-derived cardiomyocytes are both a useful model to study cardiomyogenesis in vitro and a potential source of cells for repairing failing hearts. However, enrichment of heterogeneous ES cellderived populations into cardiomyocytes remains a major challenge. Here we describe a genetic selection system based on bicistronic expression vectors that contain both the bsd resistance gene and an EGFP reporter gene expressed from the $\alpha-\mathrm{MHC}$ promoter. Selection with blasticidin $S$ resulted in a relatively pure population of ES cell-derived cardiomyocytes. Insertion of the HS2 element from the $\beta$-globin LCR upstream of the transgene promoter resulted in enhanced EGFP expression in ES cell lines. Previous data showed that transcriptional enhancers may act in cis to suppress position-effect variegation (Walters et al., 1996). Using a stably integrated reporter gene, recombinase-mediated excision of the HS2 element caused a dramatic increase in the number of $\mathrm{K} 562$ erythroleukemia cells showing transgene silencing. This observation suggests that the HS2 element may inhibit transgene silencing. In the present study, insertion of the HS2 element in the EFI-MLC3f-EGFP expression vector significantly enhanced EGFP expression, as assessed by flow cytometry. EGFP was not detectable using a CMV promoter, consistent with previous data showing transcriptional activity of the EF- $1 \alpha$, but not the CMV, promoter in naïve ES cells and EBs (Chung et al., 2002). A peculiar feature of our selection system is the use of a fusion protein consisting of sarcomeric $\alpha$-actinin protein and EGFP for epitope tagging. The newly synthesized EGFP fusion protein was correctly incorporated at the Zband of the sarcomeres, suggesting that the exogenous protein was assembled into myofibrils in ES cell-derived cardiomyocytes using the same molecular interactions that govern assembly of the corresponding endogenous protein. Therefore, this system may be applicable to transgenic mouse models where it may allow characterization of mutant proteins involved in cardiomyopathies. However, sarcomeric localization was lost using the MLC3f-EGFP fusion protein expressed from the $\alpha-\mathrm{MHC}$ promoter, despite being maintained using the MLC-2v, NCXI and $\mathrm{CTnI}$ promoters. The reason for this promoter-specific effect is unclear. An additional aspect of novelty of the present selection system is the use of the bsd gene from Aspergillus terreus (Kimura et al., 1994). This results in faster selection (3 days), as compared to other systems including neomycin or puromycin resistance genes (6-8 days) (Klug et al., 1996). No toxic effects of blasticidin S on ES cell-derived cardiomoycytes was apparent even after 10 days of treatment. Another practical advantage of the bsd gene is that, unlike neomycin and puromycin resistance genes, it is not part of a large number of expression vectors used for molecular cloning. In our model, blasticidin $\mathrm{S}$ selected for a relatively pure population of ES cell-derived cardiomyocytes that showed normal morphology, myofibrillar architecture, cell-cell contacts, and growth in culture. A limitation of the present study is that ES cell lines expressing EGFP fusion proteins were generated using the $\alpha-\mathrm{MHC}$, but not the MLC-2v, the NCXI or the CTnI promoter, as assessed by microscopical screening of 25 clones for each gene construct. Although low-level EGFP expression in some of these clones remains a possibility, bright green fluorescent cell clusters were not observed under the fluorescence microscope. This contrasts with our observations in transiently transfected neonatal rat cardiomyocytes, in which the MLC2V, NCXI and CTnI promoter directed MLC3fEGFP expression detectable at the microscopical level. Differences in gene expression between stable and transient transfection conditions have been observed in a number of cell systems (Makar et al., 1998), reflecting the importance of nuclear matrix or chromatin interactions, or of additional transcriptional regulatory elements outside the promoter region for appropriate gene expression. Earlier studies reported that the endogenous MLC-2v, NCXI and CTnI promoters are active on day 7 of ES cell differentiation (Boheler et al., 2005). Moreover, the MLC2v promoter (Franz et al., 1993; Meyer et al., 2000; Müller et al., 2000), the NCXI HI promoter (Müller et al., 2002), and the cTnI promoter (Opherk et al., 2007) have been shown to drive cardiac-specific transgene expression in mouse embryos. Of note, earlier patchclamp studies showed that EGFP expression driven by the $\alpha-\mathrm{MHC}$ promoter is restricted to pacemaker and atrial, but does not include ventricular, ES cell-derived cardiomyocytes (Kolossov et al., 2005). In contrast, the MLC-2v promoter allows selection of cardiomyocytes with ventricular specification (Meyer et al., 2000; Müller et al., 2000). In conclusion, we have described a genetic system that allows rapid and effective selection of ES cell-derived cardiomyocytes and simultaneous monitoring of myofibrillogenesis in differentiating myocytes by vital fluorescence microscopy. 


\section{Acknowledgments}

The authors thank Dr. A. Nagy (Toronto, Canada) for R1 ES cells, Dr. J. Gulick (Cincinnati, OH, USA) for plasmid aMHC-clone26, Dr. Y. Jin (Winnipeg, Canada) for the MLCp.luc construct, Dr. J. G. Müller (Charleston, SC, USA) for the pGL2-Basic Vector+2.6 Kb NCX1 promoter construct, Dr. P. Barton (London, UK) for the "-463troponinI" construct, Dr. M. Gautel (London, UK) for plasmid pET6-ACTN2, and Dr. I. Agarkova (Zürich, Switzerland) for EH-myomesin antibodies. Drs. D. Auerbach, A. Hirschy, I. Agarkova, and Ms. Sara Meier are gratefully acknowledged for scientific and technical support. R.B. was supported by pre-doctoral training grants by the ETH Zürich, the Swiss Cardiovascular Training and Research Network, the Swiss University Conference, and the Teo Rossi di Montelera Foundation, as well as by grant 31-63486 by the Swiss National Science Foundation and grant P-038-01 by the Gebert-Rüf Stiftung.

\section{References}

Auerbach D, Rothen-Ruthishauser B, Bantle S, Leu M, Ehler E, Helfman D, et al. Molecular mechanisms of myobril assembly in heart. Cell Struct Funct 1997;22:139-46.

Bhavsar PK, Dellow KA, Yacoub MH, Brand NJ, Barton PJ. Identification of cis-acting DNA elements required for expression of the human cardiac troponin I gene promoter. J Mol Cell Cardiol 2000;32:95-108.

Boer $\mathrm{PH}$. Activation of the gene for type-b natriuretic factor in mouse stem cell cultures induced for cardiac myogenesis. Biochem Biophys Res Commun 1994;199:954-61.

Boheler KR, Crider DG, Tarasova Y, Maltsev VA. Cardiomyocytes derived from embryonic stem cells. Methods Mol Med 2005;108:417-35.

Bulger M, van Doorninck JH, Saitoh N, Telling A, Farrell C, Bender MA, et al. Conservation of sequence and structure flanking the mouse and human beta-globin loci: the beta-globin genes are embedded within an array of odorant receptor genes. Proc Natl Acad Sci USA 1999;96:5129-34.

Chung S, Andersson T, Sonntag K-C, Björklund L, Isacson 0, Kin K-S. Analysis of different promoter systems for efficient transgene expression in mouse embryonic stem cell lines. Stem Cells 2002;20:139-45.

Dätwyler DA, Magyar JP, Busceti V, Hirschy A, Perriard JC, Bailey JE, et al. Recombinant Sindbis virus allows expression and precise targeting of proteins of the contractile apparatus in cultured cardiomyocytes. Basic Res Cardiol 2001;96:630-5.

Ehler E, Rothen BM, Hammerle SP, Komiyama M, Perriard JC. Myofibrillogenesis in the developing chicken heart: assembly of Z-disk, Mline and the thick filaments. J Cell Sci 1999;112:1529-39.

Erdo F, Buhrle C, Blunk J, Hoehn M, Xia Y, Fleischmann B, et al. Host-dependent tumorigenesis of embryonic stem cell transplantation in experimental stroke. J Cereb Blood Flow Metab 2003;23:780-5.

Fijnvandraat AC, an Ginneken ACG, de Boer PAJ, Ruijter JM, Christoffels VM, Moorman AFM, et al. Cardiomyocytes derived from embryonic stem cells resemble cardiomyoyctes of the embryonic heart tube. Cardiovasc Res 2003:58:399-409.

Franz WM, Breves D, Klingel K, Brem G, Hofschneider PH, Kandolf R. Heartspecific targeting of firefly luciferase by the myosin light chain-2 promoter and developmental regulation in transgenic mice. Circ Res 1993;73:62938.

Ganim JR, Luo W, Ponniah S, Grupp I, Kim HW, Ferguson DG, et al. Mouse phospholamban gene expression during development in vivo and in vitro. Circ Res 1992;71:1021-30.

Grosveld F. Activation by locus control regions? Curr Opin Genet Dev 9:152-7.

Grove BK, Kurer V, Lehner C, Doetschman TC, Perriard J-C, Eppenberger HM. A new 185,000 Dalton skeletal muscle protein detected by monoclonal antibodies. J Cell Biol 1999;98:518-24.

Gulick J, Subramaniam A, Neumann J, Robbins J. Isolation and characterization of the mouse cardiac myosin heavy chain genes. J Biol Chem 1991;266:9180-5.

Hidaka K, Lee J-K, Kim HS, Ihm CH, Iio A, Ogawa M, et al. Chamber-specif- ic differentiation of $\mathrm{Nkx2.5-positive} \mathrm{cardiac} \mathrm{precursor} \mathrm{cells} \mathrm{from} \mathrm{murine}$ embryonic stem cells. FASEB J 2003;17:740-2.

Huber I, Itzhaki I, Caspi 0, Arbel G, Tzukerman M, Gepstein A, et al. Identification and selection of cardiomyocytes during human embryonic stem cell differentiation. FASEB J 2007;21:2551-63.

Jin $Y$, Pasumarthi KB, Bock ME, Chen $Y$, Kardami E, Cattini PA. Effect of "enhancer" sequences on ventricular myosin light chain-2 promoter activity in heart muscle and nonmuscle cells. Biochem Biophys Res Commun 1995;210:260-6.

Kehat I, Kenyagin-Karsenti D, Snir M, Segev H, Amit M, Gepstein A, et al. Human embryonic stem cells can differentiate into myocytes with structur$\mathrm{al}$ and functional properties of cardiomyocytes. J Clin Invest 2001;108:407-14.

Kimura M, Takatsuki A, Yamaguchi I. Blasticidin S deaminase gene from Aspergillus terreus (BSD): a new drug resistance gene for transfection of mammalian cells. Biochim Biophys Acta 1994;1219:653-9.

Klug MG, Soonpaa MH, Koh GY, Field LJ. Genetically selected cardiomyocytes from differentiating embryonic cells form stable intracardiac grafts. J Clin Invest 1996;98:216-24.

Kolossov E, Fleischmann BK, Liu Q, Bloch W, Viatchenko-Karpinski S, Manzke 0 , et al. Functional characteristics of ES cell-derived cardiac precursor cells identified by tissue-specific expression of the green fluorescent protein. J Cell Biol 1998;143:2045-56.

Kolossov E, Lu Z, Drobinskaya I, Gassanov N, Duan Y, Sauer H, et al. Identification and characterization of embryonic stem cell-derived pacemaker and atrial cardiomyocytes. FASEB J 2005;19:577-9.

Makar KW, Pham CT, Dehoff MH, O'Connor SM, Jacobi SM, Holers VM. An intronic silencer regulates $B$ lymphocyte cell- and stage-specific expression of the human complement receptor type 2 (CR2, CD21) gene. J Immunol 1998;160:1268-78.

Meyer N, Jaconi M, Landopoulou A, Fort P, Pucéat M. A fluorescent reporter gene as a marker for ventricular specification in ES-derived cardiac cells. FEBS Letters 2000;478:151-8.

Miller-Hance WC, LaCorbiere M, Fuller SJ, Evans SM, Lyons G, Schmidt C, et al. In vitro chamber specification during embryonic stem cell cardiogenesis. Expression of the ventricular myosin light chain-2 gene is independent of heart tube formation. J Biol Chem 1993;268:25244-52.

Moore JC, Spijker R, Martens AC, de Boer T, Rook MB, van der Heyden MAG, et al. A P19Cl6 GFP reporter line to quantify cardiomyocyte differentiation of stem cells. Int J Dev Biol 2004;48:47-55.

Muthuchamy M, Pajak L, Howles L, Doetschman T, Wieczorek DF. Developmental analysis of tropomyosin gene expression in embryonic stem cells and mouse embryos. Mol Cell Biol 1993;13:3311-23.

Müller JG, Isomatsu Y, Koushik SV, O’Quinn M, Xu L, Kappler CS, et al. Cardiac-specific expression and hypertrophic upregulation of the feline $\mathrm{Na}(+)-\mathrm{Ca}(2+)$ exchanger gene $\mathrm{H} 1$-promoter in a transgenic mouse model. Circ Res 2002; 90:158-64.

Müller M, Fleischmann BK, Selbert S, Ji GJ, Endl E, Middeler G, et al. Selection of ventricular-like cardiomyocytes from $E S$ cells in vitro. FASEB J 2000; 14:2540-8.

Nagy A, Rossant J, Nagy R, Abramow-Newerly W, Roder JC. Derivation of completely cell culture-derived mice from early-passage embryonic stem cells. Proc Natl Acad Sci USA 1993;90:8424-8.

Opherk JP, Yampolsky P, Hardt SE, Schoels W, Katus HA, Koenen M, et al. Cardiac-specific activation of Cre expression at late fetal development. Biochem Biophys Res Commun 2007;359:209-13.

Sanchez A, Jones WK, Gulick J, Doetschman T, Robbins J. Myosin heavy chain gene expression in mouse embryoid bodies. An in vitro developmental study. J Biol Chem 1991;266:22419-26.

Sen A, Dunnmon P, Henderson SA, Gerard RD, Chien KR. Terminally differentiated neonatal rat myocardial cells proliferate and maintain specific differentiated functions following expression of SV40 large T antigen. J Biol Chem 1988;263:19132-6.

Smith AG. Mouse embryo stem cells: their identification, propagation and manipulation. Semin Cell Biol 1992;3:385-99.

Walters MC, Magis W, Fiering S, Eidemiller J, Scalzo D, Groudine M, et al. Transcriptional enhancers act in cis to suppress position-effect variegation. Genes Dev 1996;10:185-95.

Xu C, Police S, Rao N, Carpenter MK. Characterization and enrichment of cardiomyocytes derived from human embryonic stem cells. Circ Res 2002;91:501-8.

Young P, Ferguson C, Banuelos S, Gautel M. Molecular structure of the sarcomeric Z-disk: two types of titin interactions lead to an asymmetrical sorting of alpha-actinin. EMBO J 1998;17:1614-24. 